\title{
molecules
}

ISSN 1420-3049

(C) 2008 by MDPI

www.mdpi.org/molecules

Editorial

\section{7 - An Excellent Year for Molecules and a Look Forward to 2008}

\section{Derek McPhee and Shu-Kun Lin}

Molecular Diversity Preservation International (MDPI), Matthaeusstrasse 11, Basel CH-4057

Switzerland; Tel. ++41 79322 3379, Fax: ++41 61302 8918, E-mails: mcphee@mdpi.org;

lin@mdpi.org; http://www.mdpi.org/lin

Received: 3 January 2008 / Published: 9 January 2008

As already anticipated in August 2007 [1], the year that just concluded proved to be a truly outstanding one for MDPI's flagship journal Molecules. By the end of the year we had published 221 papers and six Editorials or Editor's Notes for a total of 2657 pages, which represented a 162\% increase over 2006. Largely thanks to the efforts of our Guest Editors Professors Pereira, Andrade, Valentao, Sottomayor, Kolehmainen, and Vanden Eynde, these papers included the first ones submitted for our ongoing special issues on Heterocycles, Nucleic Acid Derivatives in Organic Chemistry, Phenolics (Flavonoids and the Non-flavonoids), Bile Acids and Prodrugs: from Design to Applications. Additional papers from these issues shall continue to appear in 2008 as they complete the peer review process. In light of the success of these series we have also announced for 2008 new thematic issues on Spirocompounds, Alkaloids, Polysaccarides, Toxins, 5-Fluorouracil, Natural Product Pre-Fractionation [2] and as in previous years, we also plan to publish expanded peerreviewed versions of select papers presented at ECSOC-11, the $11^{\text {th }}$ International Electronic Conference on Synthetic Organic Chemistry (ECSOC, http://www.mdpi.org/ecsoc/).

We are proud to claim that this increase in publishing volume has not been at the expense of quality. We have continued to maintain a rigorous peer-review process and to be vigilant for unethical behaviour such as dual publication or plagiarism, as evidenced by the withdrawal during 2007 of some previously published papers. As our readership will know, we have by now moved to a fully OpenAccess format, with publication fees paid by authors or their institutions. While continuing to charge considerably less than competing publications offering a similar option, we anticipate that this move will reflect positively in our 2007 Impact Factor, as discussed in one of our editorials [3].

We have also responded to a request from many of our authors and readers to ensure that all papers published in Molecules are indexed by PubMed/Medline. This entailed a considerable effort by our staff but we are pleased to announce that all papers published since 2004 are already indexed [4] and we anticipate adding DOI numbers for all prior volumes during 2008. To accommodate the increased 
workload generated by all this activity, a stronger editorial office will be set up and a new editorial assistant will be joining our offices in Basel soon.

In conclusion, as 2007 ends and 2008 starts, we take this opportunity to offer our best wishes and thank our many authors, peer-reviewers and readers for their continued support of our publishing efforts. As always, we welcome your opinions on these matters. Messages with a suitable Subject header should be sent to lin@mdpi.org.

\section{References and Notes}

1. Lin, S.-K. Full Open Access Publishing Policy Imposed in 2007: Molecules Publishes Many More Papers This Year - More than 2000 Pages Published up to Issue 8. Molecules 2007, 12, 2001-2002 (PDF format 15K at http://www.mdpi.org/molecules/papers/12082001.pdf).

2. These new and prior special issues may be accessed from the Molecules website homepage: http://www.mdpi.org/molecules/index.htm

3. Lin, S.-K. Non-Open Access and Its Adverse Impact on Molecules. Molecules 2007, 12, 14361437 (PDF format 16K at http://www.mdpi.org/molecules/papers/12071436.pdf; HTML format at http://www.mdpi.org/molecules/html/12071436.htm).

4. The full list is accessible by clicking on the PubMed link on the Molecules homepage at http://www.mdpi.org/molecules/index.htm

(C) 2008 by Molecular Diversity Preservation International (MDPI). 